\title{
An improvement result concerning fixed point theory for cyclic contractions
}

\section{MoHAmed Jleli and Bessem SAMET}

\begin{abstract}
.
In this note, we obtain an improvement result for cyclic contractions by weakening the closure assumption that is usually supposed in the literature. We present some applications of the obtained result to prove the existence of solutions for a system of functional equations.
\end{abstract}

Acknowledgements. The second author extends his appreciation to Distinguished Scientist Fellowship Program (DSFP) at King Saud University (Saudi Arabia).

\section{REFERENCES}

[1] Agarwal, R. P., Alghamdi, M. A. and Shahzad, N., Fixed point theory for cyclic generalized contractions in partial metric spaces, Fixed Point Theory Appl., 2012:40

[2] Banach, S., Sur les opérations dans les ensembles abstraits et leur applications aux équations intégrales, Fundam. Math., 3 (1922), 133-181

[3] Berinde, V. and Petric, M. A., Fixed point theorems for cyclic non-self single-valued almost contractions, Carpathian J. Math., 31 (2015), 289-296

[4] Berinde, V. and Vetro, F., Fixed point for cyclic weak $(\psi, C)$-contractions in 0-complete partial metric spaces, Filomat., 27 (2013), 1405-1413

[5] Du, W.-S. and Karapinar, E., A note on Caristi-type cyclic maps: related results and applications, Fixed Point Theory Appl., 2013, 2013:344

[6] Karapinar, E., Best proximity points of cyclic mappings, Appl. Math. Lett., 335 (2007), 79-92

[7] Karapinar, E., Fixed point theory for cyclic weak $\varphi$-contraction, Appl. Math. Lett. 24 (2011), 822-825.

[8] Karapinar, E., Shobkolaei, N., Sedghi, S. and Vaezpour, S. M., A common fixed point theorem for cyclic operators on partial metric spaces, Filomat., 26 (2012), 407-414

[9] Kirk, W. A., Srinivasan, P. S. and Veeramani, P., Fixed points for mappings satisfying cyclical contractive conditions, Fixed Point Theory., 4 (2003), 79-89

[10] Mongkolkeha, C. and Kumam, P., Best proximity point theorems for generalized cyclic contractions in ordered metric spaces, J. Optim. Theory Appl., 155 (2012), 215-226

[11] Nashine, H. K., Cyclic generalized $\psi$-weakly contractive mappings and fixed point results with applications to integral equations, Nonlinear Anal., 75 (2012), 6160-6169

[12] Nashine, H. K., Kadelburg, Z. and Radenović, S., Fixed point theorems via various cyclic contractive conditions in partial metric spaces, Publ. de 1'Inst. Math., 93, (2013), 69-93

[13] Păcurar, M., and Rus, I. A., Fixed point theory for cyclic $\varphi$-contractions, Nonlinear Anal., 72 (2010), 1181-1187

[14] Petric, M. A., Some results concerning cyclical contractive mappings, General Mathematics., 18 (2010), 213-226

[15] Petruşel, G., Cyclic representations and periodic points, Studia Univ. Babes-Bolyai Math., 50 (2005), 107-112

[16] Radenović, S., A note on fixed point theory for cyclic $\varphi$-contractions, Fixed Point Theory Appl., (2015) 2015:189

Received: 24.12.2015; In revised form: 04.04.2016; Accepted: 15.04.2016

2010 Mathematics Subject Classification. 47H10.

Key words and phrases. Cyclic contraction, fixed point, closure.

Corresponding author: B. Samet; bsamet@ksu.edu.sa 
[17] Rus, I. A., Cyclic representations and fixed points, Ann. T. Popoviciu, Seminar Funct. Eq. Approx. Convexity., 3 (2005), 171-178

DePARTMENT OF MATHEMATICS

COLlEGE OF SCIENCE

KING SAUd UNIVERSITY

P. O. BOX 2455, RIYADH 11451, SAUDI ARABIA

E-mail address: jleli@ksu.edu.sa

E-mail address: bsamet@ksu. edu.sa 